\title{
A NOTE ON BERESTYCKI-CAZENAVE'S CLASSICAL INSTABILITY RESULT FOR NONLINEAR SCHRÖDINGER EQUATIONS
}

\author{
STEFAN LE COZ
}

\begin{abstract}
In this note we give an alternative, shorter proof of the classical result of Berestycki and Cazenave on the instability by blow-up for the standing waves of some nonlinear Schrödinger equations.
\end{abstract}

\section{INTRODUCTION}

In 1981, in a celebrated note [1], Berestycki and Cazenave studied the instability of standing waves for the nonlinear Schrödinger equation

$$
i u_{t}+\Delta u+|u|^{p-1} u=0
$$

where $u=u(t, x) \in \mathbb{C}, t \in \mathbb{R}, x \in \mathbb{R}^{N}$ and $p>1$. A standing wave is a solution of (1) of the form $e^{i \omega t} \varphi(x)$ with $\varphi \in H^{1}\left(\mathbb{R}^{N}\right)$ and $\omega>0$. Thus $\varphi$ is solution of

$$
-\Delta \varphi+\omega \varphi=|\varphi|^{p-1} \varphi, \quad \varphi \in H^{1}\left(\mathbb{R}^{N}\right)
$$

We say that $\varphi \in H^{1}\left(\mathbb{R}^{N}\right)$ is a ground state solution of $(2)$ if it satisfies

$$
\tilde{S}(\varphi)=\inf \left\{\tilde{S}(v) ; v \in H^{1}\left(\mathbb{R}^{N}\right) \backslash\{0\} \text { is a solution of } 2\right\}
$$

where $\tilde{S}$ is defined for $v \in H^{1}\left(\mathbb{R}^{N}\right)$ by

$$
\tilde{S}(v):=\frac{1}{2}\|\nabla v\|_{2}^{2}+\frac{\omega}{2}\|v\|_{2}^{2}-\frac{1}{p+1} \int_{\mathbb{R}^{N}}|v|^{p+1} d x .
$$

In [1] it is shown that if $1+\frac{4}{N}<p<1+\frac{4}{N-2}$ when $N \geqslant 3$ and $1+\frac{4}{N}<p<+\infty$ when $N=1,2$, then any standing wave associated with a ground state solution $\varphi$ of $(2)$ is unstable by blow up. More precisely, there exists $\left(\varphi_{n}\right) \subset H^{1}\left(\mathbb{R}^{N}\right)$ such that $\varphi_{n} \rightarrow \varphi$ in $H^{1}\left(\mathbb{R}^{N}\right)$ and the corresponding maximal solution $u_{n}$ of (1) with $u_{n}(0)=\varphi_{n}$ blows up in finite time.

The result and perhaps more the methods introduced in [1] still have a deep influence on the field of instability for nonlinear Schrödinger and related equations. In particular the idea of defining appropriate invariant sets and

Date: October 29, 2018.

2000 Mathematics Subject Classification. 35Q55,(35B35,35A15). 
how to use them to establish the blow-up. We should mention that in [1] more general nonlinearities were considered. The paper [1] is only a short note which contains the main ideas but no proofs. For the special nonlinearity $|u|^{p-1} u$ these proofs can be found in [5]. For the general case it seems that the extended version [2] of [1] has remained unpublished so far.

The aim of the present note is to present an alternative, shorter proof of the result of [1] for general nonlinearities. Also the instability of the standing waves is proved under slightly weaker assumptions. Before stating our result we need to introduce some notations. Let $g: \mathbb{R} \mapsto \mathbb{R}$ be an odd function extended to $\mathbb{C}$ by setting $g(z)=g(|z|) z /|z|$ for $z \in \mathbb{C} \backslash\{0\}$. Equation (1) now becomes

$$
i u_{t}+\Delta u+g(u)=0
$$

and correspondingly for the ground states we have

$$
-\Delta \varphi+\omega \varphi=g(\varphi) \text {. }
$$

For $z \in \mathbb{C}$ let $G(z):=\int_{0}^{|z|} g(s) d s$. We assume

$\left(A_{0}\right)$ The function $g$ satisfies

(a) $g \in \mathcal{C}(\mathbb{R}, \mathbb{R})$.

(b) $\lim _{s \rightarrow 0} \frac{g(s)}{s}=0$.

(c) when $N \geqslant 3, \lim _{s \rightarrow+\infty} g(s) s^{-\frac{N+2}{N-2}}=0$;

when $N=2$, for any $\alpha>0$, there exists $C_{\alpha}>0$ such that $|g(s)| \leqslant C_{\alpha} e^{\alpha s^{2}}$ for all $s>0$.

$\left(A_{1}\right)$ The function $h(s):=(s g(s)-2 G(s)) s^{-(2+4 / N)}$ is strictly increasing on $(0,+\infty)$ and $\lim _{s \rightarrow 0} h(s)=0$.

$\left(A_{2}\right)$ There exist $C>0$ and $\alpha \in\left[0, \frac{4}{N-2}\right)$ if $N \geqslant 3, \alpha \in[0, \infty)$ if $N=2$, such that

$$
|g(s)-g(t)| \leqslant C\left(1+|s|^{\alpha}+|t|^{\alpha}\right)|t-s|
$$

for all $s, t \in \mathbb{R}$. If $N=1$ we assume that for every $M>0$, there exists $L(M)>0$ such that

$$
|g(s)-g(t)| \leqslant L(M)|s-t|
$$

for all $s, t \in \mathbb{R}$ such that $|s|+|t| \leqslant M$.

Finally we define for $v \in H^{1}\left(\mathbb{R}^{N}\right)$ the functional

$$
S(v):=\frac{1}{2}\|\nabla v\|_{2}^{2}+\frac{\omega}{2}\|v\|_{2}^{2}-\int_{\mathbb{R}^{N}} G(v) d x
$$

and set

$$
m:=\inf \left\{S(v) ; v \in H^{1}\left(\mathbb{R}^{N}\right) \backslash\{0\} \text { is a solution of (4) }\right\} .
$$


Our main result is

Theorem 1. Assume that $\left(A_{0}\right)-\left(A_{2}\right)$ hold and let $\varphi$ be a ground state solution of (4), i.e. a solution of (4) such that $S(\varphi)=m$. Then for every $\varepsilon>0$ there exists $u_{0} \in H^{1}\left(\mathbb{R}^{N}\right)$ such that $\left\|u_{0}-\varphi\right\|_{H^{1}\left(\mathbb{R}^{N}\right)}<\varepsilon$ and the solution $u$ of (3) with $u(0)=u_{0}$ satisfies

$$
\lim _{t \rightarrow T_{u_{0}}}\|\nabla u(t)\|_{2}=+\infty \text { with } T_{u_{0}}<+\infty .
$$

From [3, 4] we know that assumption $\left(A_{0}\right)$ is almost necessary to guarantee the existence of a solution for (4). Assumption $\left(A_{1}\right)$ is a weaker version of the assumption (H.1) in [1. An assumption of this type, on the growth of $g$, is necessary since it is known from [6] that when $g(u)=|u|^{p-1} u$ with $1<p<1+\frac{4}{N}$ the standing waves associated with the ground states are, on the contrary, orbitally stable. Assumption $\left(A_{2}\right)$ is purely technical and is aimed at ensuring the local well-posedness of the Cauchy problem for (3). It replaces assumption (H.2) in [1]. Indeed, in [1] the authors were using the results of Ginibre and Velo [8] for that purpose. Since [1] has been published, advances have been done in the study of the Cauchy problem (see [5, 7] and the references therein). In particular, under our condition $\left(A_{2}\right)$, for all $u_{0} \in H^{1}\left(\mathbb{R}^{N}\right)$ there exist $T_{u_{0}}>0$ and a unique solution $u \in \mathcal{C}\left(\left[0, T_{u_{0}}\right), H^{1}\left(\mathbb{R}^{N}\right)\right) \cap \mathcal{C}^{1}\left(\left[0, T_{u_{0}}\right), H^{-1}\left(\mathbb{R}^{N}\right)\right)$ of $(3)$ such that $\lim _{t \rightarrow T_{u_{0}}}\|\nabla u(t)\|_{2}=+\infty$ if $T_{u_{0}}<+\infty$. Furthermore, the following conservation properties hold : for all $t \in\left[0, T_{u_{0}}\right)$ we have

$$
\begin{aligned}
S(u(t)) & =S\left(u_{0}\right), \\
\|u(t)\|_{2} & =\left\|u_{0}\right\|_{2} .
\end{aligned}
$$

Finally, the function $f: t \mapsto\|x u(t)\|_{2}^{2}$ is $\mathcal{C}^{2}$ and we have the virial identity

$$
\partial_{t t} f(t)=8 Q(u(t))
$$

where $Q$ is defined for $v \in H^{1}\left(\mathbb{R}^{N}\right)$ by

$$
Q(v):=\|\nabla v\|_{2}^{2}-\frac{N}{2} \int_{\mathbb{R}^{N}}(g(|v|)|v|-2 G(v)) d x .
$$

The proofs of instability in [1] and here share some elements, in particular the introduction of sets invariant under the flow. The main difference lies in the variational characterization of the ground states which is used to define the invariant sets and how to derive this characterization.

In [1] it is shown that a ground state of (4) can be characterized as a minimizer of $S$ on the constraint

$$
M:=\left\{v \in H^{1}\left(\mathbb{R}^{N}\right) \backslash\{0\}, Q(v)=0\right\} .
$$


To show this characterization, $S$ is directly minimized on $M$. Additional assumptions (see (H.1) in [1]) are necessary at this step to insure that the minimizing sequences are bounded. Once the existence of a minimizer for $S$ on $M$ has been established, one has to get rid of the Lagrange multiplier, namely to prove that it is zero. There, a stronger version of $\left(A_{0}\right)$, requiring in particular $g \in \mathcal{C}^{1}(\mathbb{R}, \mathbb{R})$ and a control on $g^{\prime}(s)$ at infinity, is necessary along with tedious calculations.

Having established that the ground states of (4) minimize $S$ on $M$, Berestycki and Cazenave show that the set

$$
K:=\left\{v \in H^{1}\left(\mathbb{R}^{N}\right), S(v)<m \text { and } Q(v)<0\right\}
$$

is invariant under the flow of (3) and that one can choose in $K$ an initial data, arbitrarily close to the ground state, for which the blow-up occurs.

In our approach we characterize the ground states as minimizers of $S$ on

$$
\mathscr{M}:=\left\{v \in H^{1}\left(\mathbb{R}^{N}\right) \backslash\{0\} ; Q(v)=0, I(v) \leqslant 0\right\},
$$

where $I(v)$ is defined for $v \in H^{1}\left(\mathbb{R}^{N}\right)$ by

$$
I(v):=\|\nabla v\|_{2}^{2}+\omega\|v\|_{2}^{2}-\int_{\mathbb{R}^{N}} g(|v|)|v| d x
$$

and correspondingly our invariant set is

$$
\left\{v \in H^{1}\left(\mathbb{R}^{N}\right), S(v)<m, Q(v)<0 \text { and } I(v)<0\right\} .
$$

The dominant feature of our approach, which also explains why our assumptions on $g$ are weaker than in [1] is that we never explicitly solve a minimization problem. At the heart of our approach is an additional characterization of the ground states as being at a mountain pass level for $S$. This characterization was derived in [10] for $N \geqslant 2$ and in [11] for $N=1$. We also strongly benefit from recent techniques developed by several authors [12, 13, 14, 15, 16, 17] where minimization approches using two constraints have been introduced.

\section{Proof of Theorem 1}

We first prove the existence of ground states and the fact that they correspond to minimizers of $S$ on the Nehari manifold.

Lemma 1. Assume that $\left(A_{0}\right)$ and $\left(A_{1}\right)$ hold. Then (4) admits a ground state solution. Furthermore, the ground states solutions of (4) are minimizers for

$$
d(\omega):=\inf \left\{S(v) ; v \in H^{1}\left(\mathbb{R}^{N}\right) \backslash\{0\}, I(v)=0\right\} .
$$

Before proving Lemma 1, we prove a technical result. 
Lemma 2. Assume that $\left(A_{0}\right)$ and $\left(A_{1}\right)$ hold. Then the nonlinearity $g$ satisfies

$$
\begin{gathered}
\frac{g(s)}{s} \text { is increasing for } s>0 . \\
\frac{g(s)}{s} \rightarrow+\infty \text { as } s \rightarrow+\infty .
\end{gathered}
$$

Proof of Lemma 2. From the definition of $h(s)$ we have

$$
\frac{g(s)}{s}=s^{4 / N} h(s)+\frac{2 G(s)}{s^{2}} .
$$

Furthermore, for $s>0$

$$
\frac{\partial}{\partial s}\left(\frac{G(s)}{s^{2}}\right)=\frac{s(s g(s)-2 G(s))}{s^{4}}>0
$$

where the last inequality follows from $\left(A_{1}\right)$. Thus, combining $(10),(11)$ and $\left(A_{1}\right)$ we get (8) and (9).

Proof of Lemma 1. It follows from Lemma 2 that

(P) There exists $s_{0}>0$ such that

$$
\begin{aligned}
& \text { - if } N \geqslant 2 \text {, then } \frac{1}{2} \omega s_{0}^{2}<G\left(s_{0}\right) \text {; } \\
& \text { - if } N=1 \text {, then } \frac{1}{2} \omega s^{2}>G(s) \text { for } s \in\left(0, s_{0}\right), \frac{1}{2} \omega s_{0}^{2}=G\left(s_{0}\right) \text { and } \\
& \quad \omega s_{0}<g\left(s_{0}\right) \text {. }
\end{aligned}
$$

Now, from [3, Théorème 1] and [4, Theorem 1] we know that the conditions $\left(A_{0}\right)$ and $(\mathrm{P})$ are sufficient to insure the existence of a ground state.

If $v$ is a solution of (4), then $S^{\prime}(v) v=I(v)=0$; therefore, to prove the lemma it is enough to show that $d(\omega) \geqslant m$. From [10, 11] we know that under $\left(A_{0}\right)$ and $(\mathrm{P})$ the functional $S$ has a mountain pass geometry. More precisely, if we set

$$
\Gamma:=\left\{\chi \in \mathcal{C}\left([0,1], H^{1}\left(\mathbb{R}^{N}\right)\right) ; \chi(0)=0, S(\chi(1))<0\right\},
$$

then $\Gamma \neq \emptyset$ and

$$
c:=\inf _{\chi \in \Gamma} \max _{t \in[0,1]} S(\chi(t))>0 .
$$

In addition it is shown ${ }^{1}$ in [10, 11] that

$$
m=c .
$$

Namely the mountain pass level c corresponds to the ground state level $\mathrm{m}$. Now it is well-known that (8) ensure that if $v \in H^{1}\left(\mathbb{R}^{N}\right)$ satisfies $I(v)=0$ then $t \mapsto S(t v)$ achieves its unique maximum on $[0,+\infty)$ at $t=1$. Also (9)

\footnotetext{
${ }^{1}$ In fact, the results of [10, 11 are proved only for real valued functions; however, it is not hard to see that they can be extended to the complex case (see [9, Lemma 14]).
} 
shows that $\lim _{t \rightarrow+\infty} S(t v)=-\infty$. From the definition of $c$, it implies that $c \leqslant S(v)$ for all $v \in H^{1}\left(\mathbb{R}^{N}\right)$ such that $I(v)=0$. Hence we have

$$
d(\omega) \geqslant c,
$$

and combined with the fact that $m=c$ it ends the proof.

Now we investigate the behavior of the functionals under some rescaling

Lemma 3. Assume that $\left(A_{0}\right)$ and $\left(A_{1}\right)$ hold. For $\lambda>0$ and $v \in H^{1}\left(\mathbb{R}^{N}\right)$, we define $v^{\lambda}(\cdot):=\lambda^{\frac{N}{2}} v(\lambda \cdot)$. We suppose $Q(v) \leqslant 0$. Then there exists $\lambda_{0} \leqslant 1$ such that

(i) $Q\left(v^{\lambda_{0}}\right)=0$,

(ii) $\lambda_{0}=1$ if and only if $Q(v)=0$,

(iii) $\frac{\partial}{\partial \lambda} S\left(v^{\lambda}\right)>0$ for $\lambda \in\left(0, \lambda_{0}\right)$ and $\frac{\partial}{\partial \lambda} S\left(v^{\lambda}\right)<0$ for $\lambda \in\left(\lambda_{0},+\infty\right)$,

(iv) $\lambda \mapsto S\left(v^{\lambda}\right)$ is concave on $\left(\lambda_{0},+\infty\right)$,

(v) $\frac{\partial}{\partial \lambda} S\left(v^{\lambda}\right)=\frac{1}{\lambda} Q\left(v^{\lambda}\right)$.

Proof of Lemma 3. Easy computations lead to

$$
\begin{aligned}
\frac{\partial}{\partial \lambda} S\left(v^{\lambda}\right) & =\frac{1}{\lambda} Q\left(v^{\lambda}\right) \\
& =\lambda\left(\|\nabla v\|_{2}^{2}-\frac{N}{2} \int_{\mathbb{R}^{N}} \lambda^{-(N+2)}\left(\lambda^{\frac{N}{2}} g\left(\lambda^{\frac{N}{2}}|v|\right)|v|-2 G\left(\lambda^{\frac{N}{2}} v\right)\right) d x\right),
\end{aligned}
$$

and recalling from $\left(A_{1}\right)$ that the function $h(s):=(s g(s)-2 G(s)) s^{-(2+4 / N)}$ is strictly increasing on $[0,+\infty)$, (i), (ii), (iii) and (v) follow easily. To see (iv), we remark that since

$$
\left(\|\nabla v\|_{2}^{2}-\frac{N}{2} \int_{\mathbb{R}^{N}} \lambda^{-(N+2)}\left(\lambda^{\frac{N}{2}} g\left(\lambda^{\frac{N}{2}}|v|\right)|v|-2 G\left(\lambda^{\frac{N}{2}} v\right)\right) d x\right)<0
$$

on $\left(\lambda_{0},+\infty\right)$, we infer from $\left(A_{1}\right)$ that $\frac{\partial}{\partial \lambda} S\left(v^{\lambda}\right)$ is strictly decreasing on $\left(\lambda_{0},+\infty\right)$, which implies (iv).

Proof of Theorem 1. We recall that

$$
\mathscr{M}=\left\{v \in H^{1}\left(\mathbb{R}^{N}\right) \backslash\{0\} ; Q(v)=0, I(v) \leqslant 0\right\},
$$

and define

$$
d_{\mathscr{M}}:=\inf \{S(v) ; v \in \mathscr{M}\} .
$$

We proceed in three steps.

STEP 1. Let us prove $d(\omega)=d_{\mathscr{M}}$. Since the ground states $\varphi$ satisfy $Q(\varphi)=I(\varphi)=0$, we have $\varphi \in \mathscr{M}$. Combined with $S(\varphi)=d(\omega)$, this implies $d_{\mathscr{M}} \leqslant d(\omega)$. Conversely, let $v \in \mathscr{M}$. If $I(v)=0$, then trivially $S(v) \geqslant d(\omega)$, 
thus we suppose $I(v)<0$. We use the rescaling defined in Lemma 3 : for $\lambda>0$ we have

$$
I\left(v^{\lambda}\right)=\lambda^{2}\|\nabla v\|_{2}^{2}+\omega\|v\|_{2}^{2}-\int_{\mathbb{R}^{N}} \lambda^{-N / 2} g\left(\lambda^{N / 2}|v|\right)|v| d x .
$$

It follows from $\left(A_{0}\right)$-(b) that $\lim _{\lambda \rightarrow 0} I\left(v^{\lambda}\right)=\omega\|v\|_{2}^{2}$ and thus by continuity there exists $\lambda_{1}<1$ such that $I\left(v^{\lambda_{1}}\right)=0$. Thus $S\left(v^{\lambda_{1}}\right) \geqslant d(\omega)$. Now, from $Q(v)=0$ and (iii) in Lemma 3 we have

$$
S(v) \geqslant S\left(v^{\lambda_{1}}\right) \geqslant d(\omega),
$$

hence $d_{\mathscr{M}}=d(\omega)$.

STEP 2. For $\lambda>0$, we set $u^{\lambda}:=\varphi^{\lambda}$. For $\lambda>1$ close to 1 , we have

$$
\begin{aligned}
& S\left(u^{\lambda}\right)<S(\varphi) \text { and } Q\left(u^{\lambda}\right)<0, \\
& I\left(u^{\lambda}\right)<0 .
\end{aligned}
$$

Indeed, (12) follows from (iii) and (v) in Lemma 3. For (13), we write

$$
\begin{aligned}
I\left(u^{\lambda}\right) & =2 S\left(u^{\lambda}\right)+\frac{2}{N} Q\left(u^{\lambda}\right)-\frac{2}{N}\left\|\nabla u^{\lambda}\right\|_{2}^{2} \\
& \leqslant 2 S(\varphi)+\frac{2}{N} Q(\varphi)-I(\varphi)-\frac{2 \lambda^{2}}{N}\|\nabla \varphi\|_{2}^{2} \\
& \leqslant \frac{2\left(1-\lambda^{2}\right)}{N}\|\nabla \varphi\|_{2}^{2}<0 .
\end{aligned}
$$

Let $u(t)$ be the solution of $(3)$ with $u(0)=u^{\lambda}$. We claim that the properties described in (12), (13) are invariant under the flow of (3). Indeed, since from (5) we have for all $t>0$

$$
S(u(t))=S\left(u^{\lambda}\right)<S(\varphi),
$$

we infer that $I(u(t)) \neq 0$ for any $t \geqslant 0$, and by continuity we have $I(u(t))<0$ for all $t \geqslant 0$. It follows that $Q(u(t)) \neq 0$ for any $t \geqslant 0$ (if not $u(t) \in \mathscr{M}$ and thus $S(u(t)) \geqslant S(\varphi)$ which contradicts (14)), and by continuity we have $Q(u(t))<0$ for all $t \geqslant 0$. Thus for all $t>0$ we have

$$
S(u(t))<S(\varphi), I(u(t))<0 \text { and } Q(u(t))<0 .
$$

STEP 3. We fix $t>0$ and define $v:=u(t)$. For $\beta>0$, let $v^{\beta}(x):=\beta^{\frac{N}{2}} v(\beta x)$. From STEP 2 we have $Q(v)<0$, thus from Lemma 3 there exists $\beta_{0}<1$ such that $Q\left(v^{\beta_{0}}\right)=0$. If $I\left(v^{\beta_{0}}\right) \leqslant 0$, we keep $\beta_{0}$, otherwise we replace it by $\tilde{\beta}_{0} \in\left(\beta_{0}, 1\right)$ such that $I\left(v^{\tilde{\beta_{0}}}\right)=0$. Thus in any case we have

$$
S\left(v^{\beta_{0}}\right) \geqslant d(\omega)
$$


and $Q\left(v^{\beta_{0}}\right) \leqslant 0$. Now from (iv) in Lemma 3 , we have

$$
S(v)-S\left(v^{\beta_{0}}\right) \geqslant\left(1-\beta_{0}\right) \frac{\partial}{\partial \beta} S\left(v^{\beta}\right)_{\mid \beta=1} .
$$

Thus, from (v) in Lemma 3, $Q(v)<0$ and $\beta_{0}<1$, we get

$$
S(v)-S\left(v^{\beta_{0}}\right) \geqslant Q(v) .
$$

Combined with (15), this gives

$$
Q(v) \leqslant S(v)-d(\omega):=-\delta<0
$$

where $\delta$ is independent of $t$ since $S$ is a conserved quantity.

To conclude, it suffices to observe that thanks to (7) and (16) we have

$$
\|x u(t)\|_{2}^{2} \leqslant-\delta t^{2}+C t+\left\|x u^{\lambda}\right\|_{2}^{2},
$$

and since the right hand side of (17) becomes negative when $t$ grows up, we easily deduce that $T_{u^{\lambda}}<+\infty$ and $\lim _{t \rightarrow T_{u^{\lambda}}}\|\nabla u(t)\|_{2}=+\infty$.

Acknowledgements. The author is grateful to Louis Jeanjean for helpful advice during the writing of this paper. He also wishes to thank Thierry Cazenave for sharing with him a digitalized version of the unpublished paper [2].

\section{REFERENCES}

[1] H. Berestycki And T. CAzenave, Instabilité des états stationnaires dans les équations de Schrödinger et de Klein-Gordon non linéaires, C. R. Acad. Sci. Paris 293, (1981), 489-492.

[2] H. Berestycki AND T. CAzEnAVE, Instabilité des états stationnaires dans les équations de Schrödinger et de Klein-Gordon non linéaires, Publications du Laboratoire d'Analyse Numérique, Université de Paris VI.

[3] H. Berestycki, T. Gallouet and O. Kavian, Équations de champs scalaires euclidiens non linéaires dans le plan, C. R. Acad. Sci. Paris 297, (1983), 307-310.

[4] H. Berestycki And P.L. Lions, Nonlinear scalar field equations I, Arch. Ration. Mech. Anal., 82, (1983), 313-346.

[5] T. Cazenave, Semilinear Schrödinger equations, Courant Lecture Notes in Mathematics, 10, (2003).

[6] T. Cazenave And P.L. Lions, Orbital stability of standing waves for some nonlinear Schrödinger equations, Comm. Math. Phys., 85, 4, (1982), 549-561.

[7] T. Cazenave And F.B. WeissleR, The Cauchy problem for the nonlinear Schrödinger equation in $H^{1}$, Manuscripta Math. 61, (1988), 477-494.

[8] J. Ginibre And G. Velo, On a class of nonlinear Schrödinger equations. I. The Cauchy problem, general case, J. Func. Anal. 32, 1, (1979), 1-32.

[9] L. JEANJEAn AND S. LE Coz, Instability for standing waves of nonlinear Klein-Gordon equations via mountain-pass arguments, preprint.

[10] L. JEANJEAN AND K. TANAKA, A note on a mountain pass characterization of least energy solutions, Adv. Nonlinear Stud. 3, 4, (2003), 445-455. 
[11] L. Jeanjean and K. Tanaka, A remark on least energy solutions in $R^{N}$, Proc. Amer. Math. Soc. 131, 8, (2003), 2399-2408.

[12] S. Le Coz, R. Fukuizumi, G. Fibich, B. Ksherim and Y. Sivan, Instability of bound states of a nonlinear Schrödinger equation with a Dirac potential, preprint.

[13] Y. LiU, Blow up and instability of solitary-wave solutions to a generalized KadomtsevPetviashvili equation, Trans. Amer. Math. Soc. 353, 1, (2001), 191-208.

[14] Y. LIU, Strong instability of solitary-wave solutions to a Kadomtsev-Petviashvili equation in three dimensions, J. Differential Equations 180, 1, (2002), 153-170.

[15] Y. LiU, X.-P. WANG AND K. WANG, Instability of standing waves of the Schrödinger equation with inhomogeneous nonlinearity, Trans. Amer. Math. Soc. 358, (2006), 21052122.

[16] M. Ohta And G. Todorova, Strong instability of standing waves for nonlinear KleinGordon equations, Discrete Contin. Dyn. Syst. 12, 2, (2005), 315-322.

[17] J. ZhANG, Sharp threshold for blowup and global existence in nonlinear Schrödinger equations under a harmonic potential, Comm. Partial Differential Equations, 30, 10-12, (2005), 1429-1443.

(Stefan LE COZ) Laboratoire de Mathématiques, Université de Franche-Comté, 25030 Besançon, FRANCE

E-mail address: slecoz@univ-fcomte.fr 